\title{
Evaluation of MRI/Ultrasound Fusion-Guided Prostate Biopsy Using Transrectal and Transperineal Approaches
}

\author{
Susanne Tewes, ${ }^{1}$ Inga Peters, ${ }^{2}$ Ansgar Tiemeyer, ${ }^{2}$ Matti Peperhove,, Dagmar Hartung, \\ Stefanie Pertschy, ${ }^{1}$ Markus A. Kuczyk, ${ }^{2}$ Frank Wacker, ${ }^{1}$ and Katja Hueper ${ }^{1}$ \\ ${ }^{1}$ Department of Diagnostic and Interventional Radiology, Hannover Medical School, Carl-Neuberg Str. 1, 30625 Hannover, Germany \\ ${ }^{2}$ Department of Urology and Urologic Oncology, Hannover Medical School, Carl-Neuberg Str. 1, 30625 Hannover, Germany
}

Correspondence should be addressed to Susanne Tewes; tewes.susanne@mh-hannover.de

Received 8 May 2017; Revised 12 August 2017; Accepted 20 August 2017; Published 28 September 2017

Academic Editor: Charnita M. Zeigler-Johnson

Copyright (C) 2017 Susanne Tewes et al. This is an open access article distributed under the Creative Commons Attribution License, which permits unrestricted use, distribution, and reproduction in any medium, provided the original work is properly cited.

Purpose. To evaluate transrectal (TR) and transperineal (TP) approaches for MRI/ultrasound (MRI/US) fusion-guided biopsy to detect prostate cancer (PCa). Materials and Methods. 154 men underwent multiparametric MRI and MRI/US fusion-guided biopsy between July 2012 and October 2016. 79/154 patients were biopsied with a TR approach and 75/154 with a TP approach. MRI was retrospectively analyzed according to PI-RADS version 2. PI-RADS scores were compared with histopathological results. Descriptive statistics, accuracy, and negative and positive predictive values were calculated. Histopathological results of first, second, and third MRI targeted biopsy cores were compared to evaluate the impact of one verus multiple targeted cores. Results. Detection rates of PCa were 39\% for TR biopsy and 75\% for TP biopsy. Sensitivity/specificity for tumor detection with PI-RADS $\geq 4$ were $81 / 69 \%$ for TR biopsy and 86/84\% for TP biopsy. In 31\% for TR biopsy and 19\% for TP biopsy, PCa was found in the second or third MRI targeted biopsy core only. Conclusion. MRI/US fusion-guided biopsy may be conducted with the TR as well as the TP approach with high accuracy, giving more flexibility for diagnosis and the option for focal treatment of PCa.

\section{Introduction}

Over the last ten years, multiparametric MRI (mpMRI) of the prostate has gained rising importance in the diagnosis of prostate cancer $(\mathrm{PCa})$. With mpMRI, high accuracy for the detection of PCa has been reported $[1,2]$. PCa detection rates by mpMRI range from 80 to $100 \%$ for Gleason score (GS) > 7, from 63 to $97 \%$ for GS 7, and from 21 to $75 \%$ for GS 6 tumors [3]. MRI/ultrasound (MRI/US) fusion software systems have been evolved and tested over the last few years [4]. Increased detection rates for high-risk PCa and decreased detection rates of low-risk PCa compared to the standard transrectal ultrasound- (TRUS-) guided biopsy method have been reported [5-9]. However, there are also studies showing that MRI-guided biopsy particularly improved detection of significant cancer after previous negative biopsy $[8,10]$. International guidelines recommend TRUS-guided biopsy as standard of care for first-round biopsy, while MRI-guided biopsy could be useful in the repeat biopsy setting $[11,12]$.
Different systems for MRI/US image fusion are available, which allow for transrectal (TR) or transperineal (TP) biopsy or for both $[4,13]$. Still, there are no clear recommendations with regard to the TR or TP approach. For systematic TRUSguided biopsy, cancer detection rates are comparable for both approaches $[14,15]$.

With regard to complications, with TR prostate biopsy, the incidence rate of infection and rectal bleeding is higher [16] and lesions located in the anterior part of the prostate, particularly in high volume prostates, might be missed. With TP prostate biopsy, on the other hand, the incidence of perineal swelling is higher [16], and, usually for ensuring more patient comfort, it needs to be done in general anesthesia, holding its own risks.

Furthermore, there are still no clear recommendations if more than one targeted biopsy core per PCa suspect MRI lesion significantly increases the cancer detection rate. A recent study by Schimmoller et al. found only minor benefit when taking a second targeted biopsy core in a cohort of 290 
TABLE 1: Patient characteristics.

\begin{tabular}{lcc}
\hline Number of patients & & 154 \\
Age $($ years $)$ & Mean \pm SD & $66 \pm 8$ \\
PSA $(\mu \mathrm{g} / \mathrm{l})$ & Mean (range) & $13(0.4-101)$ \\
Number of prebiopsies & Mean (range) & $1(0-7)$ \\
Prostate volume $(\mathrm{ml})$ & Mean \pm SD & $63 \pm 38$ \\
PI-RADS score & Mean \pm SD & $4 \pm 1$ \\
Biopsy cores & Total & 1529 \\
& Mean \pm SD per patient & $10 \pm 3$ \\
\hline
\end{tabular}

PI-RADS: Prostate Imaging Reporting and Data System; PSA: prostate specific antigen; SD: standard deviation.

patients who underwent in-bore MRI-guided prostate biopsy [17].

The purpose of our study was to evaluate MRI/US fusionguided biopsy using the TP or the TR approach with the same fusion system in terms of diagnostic accuracy, PCa detection rates, and feasibility in a clinical setting.

\section{Materials and Methods}

2.1. Patients. Between July 2012 and October 2016, 154 patients with clinical suspicion of PCa underwent MRI/US fusion-guided biopsy of the prostate. Between July 2012 and January 2015, only the TR approach was performed and, until then, 62 patients underwent fusion-guided biopsy. Therefore, $40 \%$ of patients from our cohort underwent TR biopsy, irrespective of prostate size, tumor localization according to MRI, negative initial biopsies, or patients' preferences due to unavailability of the TP approach. Since February 2015, both biopsy approaches were performed at our institution. Since then, detailed information and counseling on risks and advantages of the TR and TP approaches were given to the patients under consideration of prostate size, tumor localization according to MRI, negative initial biopsies, and patients' general condition. Under consideration of this information and patients' preferences, 17 patients underwent TR biopsy and 75 patients underwent TP biopsy after February 2015. This retrospective study focuses particularly on a descriptive analysis of clinical parameters in association with the different biopsy settings. In total, 79/154 patients underwent TR biopsy and 75/154 patients underwent TP biopsy. Written informed consent was obtained from each patient for clinically indicated mpMRI and MRI/US fusion-guided biopsy. The local ethical board waived the requirement for obtaining informed consent for this retrospective analysis. 125 patients had at least one negative previous biopsy (1-7 prebiopsies), 16 patients had no previous biopsy, 11 patients were on active surveillance, and in two patients it was unknown whether a previous biopsy had been performed. Patients' characteristics are summarized in Table 1 .

2.2. Multiparametric MRI. Multiparametric MRI was acquired according to European Society of Urogenital Radiology (ESUR) guidelines [2, 18] on a 3 Tesla system (MAGNETOM Skyra or MAGNETOM Verio, Siemens
Healthcare, Erlangen, Germany) using a 6-channel or 18-channel body coil and a spine coil. In order to reduce bowel movement, all patients without contraindications received an intravenous injection of $20 \mathrm{mg}$ butylscopolamine (Buscopan $20 \mathrm{mg}$, Boehringer Ingelheim, Germany) prior to the examination. T2 turbo spin echo (TSE) sequences were acquired in transverse, sagittal, and coronal orientation. For diffusion-weighted imaging (DWI), three $b$-values $=0-50$, $400-600$, and $\geq 800 \mathrm{~s} / \mathrm{mm}^{2}$ were used. Dynamic contrastenhanced (DCE) images were acquired in transverse plane after injection of $0.1 \mathrm{mmol} / \mathrm{kg}$ body weight gadoterate meglumine (Dotarem, Guerbet, Aulnay-sous-Bois, France) at a rate of $3 \mathrm{ml} / \mathrm{s}$ using a FLASH 3D sequence with an image update rate of $9 \mathrm{~s}$. For details of MRI protocol, please see supplementary Table 1 in Supplementary Material available online at https://doi.org/10.1155/2017/2176471.

Target lesions for MRI/US fusion-guided biopsy were chosen in a clinical setting by experienced uroradiologists. Before the publication of PI-RADS version 2 by the American College of Radiology (ACR), ESUR, and AdMeTech Foundation in December 2014, lesions were scored according to PIRADS version 1 [18]; since January 2015, lesions were scored according to PI-RADS version 2 [19]. In order to homogenize data for this study, target lesions were retrospectively analyzed according to PI-RADS version 2 [19] and ADC values, prostate volume, and lesion size were documented. Lesion location was reported according to the sector map as suggested in the PI-RADS version 2 document [19].

\subsection{MRI/US Fusion-Guided and Targeted Biopsy. MRI/US} fusion-guided biopsy was performed with the BioJet ${ }^{\mathrm{TM}}$ fusion system and software (D\&K Technologies, Barum, Germany). The technical data and usage of this system have been described previously $[20,21]$. In brief, contouring of the prostate margins and the target lesions was done by a radiologist using the transverse T2 TSE-images. Organ contours were fused with real-time TRUS during the biopsy session.

Patients were informed about advantages and risks of TR and TP prostate biopsy. Indication for TR or TP biopsy was adjusted to the clinical setting under consideration of technical availability, localization of lesion, patients' profile, and patients' preferences.

TR and TP prostate biopsies were performed by two experienced urologists in the dorsal lithotomy position under antibiotic prophylaxis. Local anesthesia (periprostatic block) was performed in patients with a TR biopsy course and general anesthesia was performed for the TP approach. TR MRI/US fusion was performed using a $3 \mathrm{D}$ triplane TR ultrasound system (BK Medical, Analogic Ultrasound Group, Pro Focus, Transducer $8818,9 \mathrm{MHz}$ ) solely operating with the side-fire function. An endocavity biplane transducer (BK 8848, 9 MHz, BK Medical, Analogic Ultrasound Group) was used for the TP approach. Biopsy cores with a core length of $22 \mathrm{~mm}$ were numbered according to the radiological anatomic sector map as described in the PI-RADS version 1 [18] or version 2 [19] document. The mean number of biopsy cores with the TR approach was $8 \pm 3$ (range: $4-12$ ) cores per patient with $3 \pm 1$ (range: 1-7) cores from targeted biopsy and $5 \pm 4$ (range: $0-11$ ) cores from additional random biopsy. 


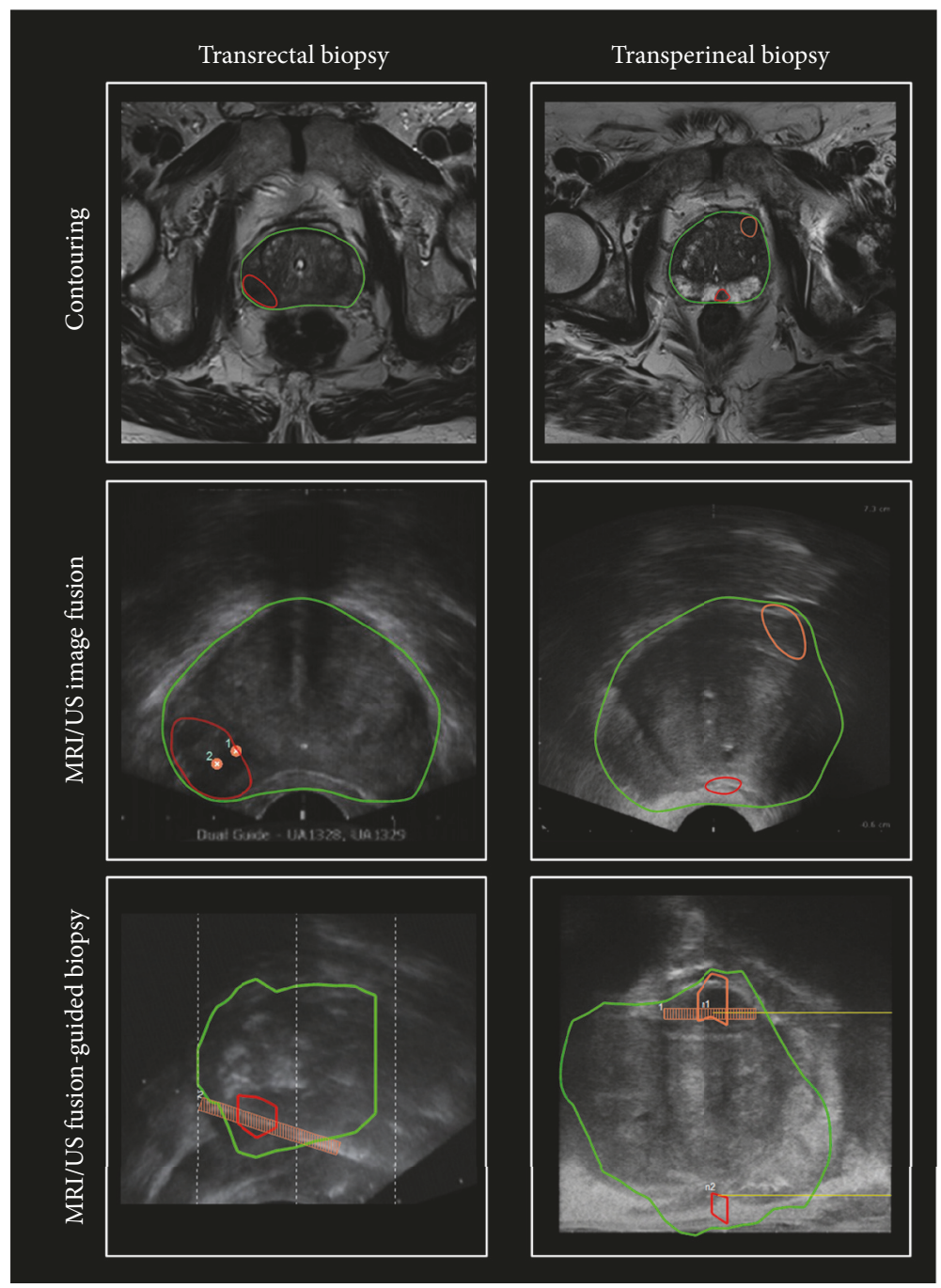

FIGURE 1: Workflow of MRI/TRUS fusion-guided biopsy with the BioJet software. Depicted are two examples of MRI/TRUS fusion-guided biopsy, one with the transrectal (left column) approach and one with the transperineal (right column) approach. First row: contouring of the prostate margins (green) and target lesions (red and orange). Second row: fusion of MRI contours and ultrasound images in the transverse orientation. Third row: obtaining samples from the target lesion. Fusion of MRI contours and ultrasound images are shown in the sagittal orientation. Biopsy cores are marked in orange.

Using the TP setting, the mean number of biopsy cores per patient was $12 \pm 1$ (range: $5-12$ ) cores with $3 \pm 1$ (range: 1-6) cores from targeted biopsy and $8 \pm 2$ (range: $2-11$ ) cores from additional random biopsy.

For each cancer-positive biopsy core, a pathologist determined the Gleason grade and Gleason score (GS). Clinically significant cancer was defined as GS $\geq 7$ and/or PSA $\geq$ $10 \mu \mathrm{g} / \mathrm{L}$, designated as intermediate and/or high-risk tumor groups according to the D'Amico criteria [22]. Examples of MRI/TRUS fusion-guided biopsy with the TR and TP approaches are shown in Figure 1.

2.4. Statistical Analysis. For statistical analysis, GraphPad Prism software version 6 (GraphPad Software, Inc., USA) and SPSS software version 24 (IBM Corporation, USA) were used. Clinical data of patients with and without biopsy-proven $\mathrm{PCa}$ as well as patients with TR and TP biopsy approaches were compared using unpaired $t$-tests and values are given as mean \pm standard deviation (SD). Descriptive analysis, sensitivity, specificity, and negative predictive value (NPV) and positive predictive value (PPV) of mpMRI in TR and TP cohorts for a PI-RADS score $\geq 4$ were evaluated with respect to the dominant lesion in each patient and analysis was performed on a per patient basis. The combined histological result of MRI/US fusion-guided and random biopsy cores served as reference.

\section{Results}

3.1. Transrectal MRI/US Fusion-Guided Prostate Biopsy (TR Cohort). In our TR cohort, 31/79 patients were diagnosed with PCa (39\%) between July 2012 and September 2016. 60/79 patients had untargeted negative prebiopsies, 6 patients were under active surveillance, 12 patients were biopsy-naïve, and 
TABLE 2: Distribution of MRI/TRUS fusion-guided biopsy target lesions and PCa in TR and TP cohorts.

\begin{tabular}{lcccc}
\hline \multirow{2}{*}{ localization } & \multicolumn{2}{c}{ Transrectal } & \multicolumn{2}{c}{ Transperineal } \\
& All lesions $(n=79)$ & Lesions with PCa* $(n=31)$ & All lesions $(n=75)$ & Lesions with PCa* $(n=56)$ \\
\hline \multirow{2}{*}{ AFS } & $9 / 79$ & $6 / 31$ & $27 / 75$ & $24 / 56$ \\
& $(11.4 \%)$ & $(19.4 \%)$ & $(36 \%)$ & $(42.9 \%)$ \\
Anterior & $18 / 79$ & $6 / 31$ & $32 / 75$ & $20 / 56$ \\
& $(22.8 \%)$ & $(19.4 \%)$ & $(42.7 \%)$ & $(35.7 \%)$ \\
Posterior & $52 / 79$ & $19 / 31$ & $16 / 75$ & $12 / 56$ \\
& $(65.8 \%)$ & $(61.2 \%)$ & $(21.3 \%)$ & $(21.4 \%)$ \\
\hline
\end{tabular}

${ }^{*}$ Lesions with tumor-positive cores after fusion-guided and systematic biopsy. AFS: anterior fibromuscular stroma; PCa: prostate cancer.

TABLE 3: Frequency of detection of low-risk, intermediate, and high-risk PCa with targeted MRI/TRUS fusion-guided and systematic biopsy.

\begin{tabular}{|c|c|c|c|c|}
\hline \multirow{2}{*}{ Risk group according to D’Amico criteria } & \multicolumn{2}{|c|}{ Transrectal } & \multicolumn{2}{|c|}{ Transperineal } \\
\hline & Targeted & Systematic & Targeted & Systematic \\
\hline Low risk & 6 & 1 & 16 & 2 \\
\hline Intermediate and high risk & 15 & 9 & 36 & 2 \\
\hline All PCa & 21 & 10 & 52 & 4 \\
\hline
\end{tabular}

The number of lesions with highest GS detected with targeted and systematic biopsy is given. The overall PCa detection rate is not shown. Highest GS were more frequently detected with targeted than with systematic biopsy with both biopsy routes. PSA: prostate specific antigen.

in one patient the prebiopsy status was unknown. One patient was excluded from the analysis because the target lesion located in the anterior part of the prostate could not be reached by TR biopsy due to restriction of the needle guide (at that time point, a TP biopsy approach was technically not available at our institution yet). 664 biopsy cores were taken in total: 271 cores were from targeted biopsy and 393 cores were from additional systematic random biopsies.

PI-RADS scores were significantly higher in patients with PCa compared to patients without $\mathrm{PCa}(4.1 \pm 0.8$ versus $2.9 \pm$ $1.0, p<0.001)$ and ADC values were significantly lower in tumor-positive lesions $\left(0.8 \pm 0.2\right.$ versus $0.9 \pm 0.310^{-3} \mathrm{~mm}^{2} / \mathrm{s}$, $p<0.01)$. Patients with PCa were significantly older $(67 \pm$ 8 versus $63 \pm 7$ years, $p<0.05)$ and prostate volume was significantly lower in the group with PCa (48 \pm 26 versus $67 \pm$ $32 \mathrm{ml}, p<0.01)$. No significant difference was found in PSA levels $(12 \pm 16$ versus $8 \pm 4 \mu \mathrm{g} / \mathrm{l})$, lesion size ( $14 \pm 6$ versus $12 \pm$ $5 \mathrm{~mm}$ ), or body mass index (BMI $28 \pm 5$ versus $27 \pm 4 \mathrm{~kg} / \mathrm{m}^{2}$ ).

According to the sector map, $27 / 79$ (33\%) target lesions from TR biopsy were located in the anterior half of the prostate; 52/79 (66\%) target lesions were in the posterior half. Concordantly, the tumor detection rate was slightly but not significantly lower in the anterior $(12 / 31,39 \%)$ than in the posterior prostate $(19 / 31,61 \%$; Table 2$)$, which is presumably solely the result of lower sampling rates in the anterior gland in this cohort. The detection rate, when only considering the samples in the respective region, was $12 / 27$ (44\%) for the anterior gland, while it was 19/52 (37\%) for the posterior gland.

In our TR cohort, sensitivity and specificity of $81 \%$ and $69 \%$ were achieved for mpMRI with a Youden-selected cutoff value of PI-RADS $\geq 4$ (area under the curve (AUC) $=0.81$ (95\% confidence interval (CI): $0.7-0.9)$ ). NPV was $85 \%$ and PPV was $63 \%$.

26 out of $31 \mathrm{PCa}$ positive biopsies were obtained by targeted biopsy (Table 3 ). The cancer detection rate was improved from $33 \%$ to $39 \%$ when additional random biopsy cores were considered: in five patients (6\%), PCa was only found in systematic biopsy (one patient with GS $3+3=6$, one patient with GS $3+4=7 \mathrm{a}$, one patient with GS $4+3=7 \mathrm{~b}$, one patient with GS $4+4=8$, and one patient with $4+5=9$ ). A retrospective reanalysis revealed that in two of these five patients no suspicious MRI lesion could be identified in the documented region of the prostate (GS: $3+3=6$ and $4+3=$ $7 \mathrm{~b})$. In the other three cases, the suspicious lesion had been identified at MRI (PI-RADS score 4 in all of the three cases), indicating that MRI/US fusion or the fusion-guided biopsy might have failed (GS: $3+4=7 \mathrm{a}, 4+4=8$, and $4+5=9$ ). Furthermore, in 5/26 patients, additional systematic biopsy cores resulted in a GS upgrade (three patients from GS $3+4=$ 7 a to $4+3=7 \mathrm{~b}$, one patient from GS $4+3=7 \mathrm{~b}$ to $4+4=8$, and one patient from GS $4+3=7 \mathrm{~b}$ to $4+5=9$; Table 3 ). A retrospective reanalysis showed that in one of these patients (GS upgrade from $4+3=7 \mathrm{~b}$ to $4+5=9$ ) initial clinical MRI report and choice of index lesion failed and the region of the prostate that revealed highest Gleason score was most suspect on MRI. In the other four cases, systematic biopsy cores that revealed Gleason score upgrade were taken from a region in the prostate that was close to the suspect lesion on MRI and might have contained prostate tissue that appeared suspect on MRI and might have been part of the index lesion.

A subgroup analysis of patients with PCa positive targeted biopsy $(n=26)$ revealed that in $18 / 26$ patients PCa was detected with the first biopsy core, in 7/26 with the second biopsy core, and in 1/26 with the third biopsy core. Thus, taking multiple biopsy cores from the target lesion improved cancer detection from $23 \%$ with one biopsy core to $32 \%$ with two and $33 \%$ with three biopsy cores using the TR approach.

3.2. Transperineal MRI/US Fusion-Guided Prostate Biopsy (TP Cohort). In the TP cohort, 56/75 patients were diagnosed with PCa (75\%) between February 2015 and September 
2016. 65/75 patients had untargeted negative prebiopsies, five patients were under active surveillance, four patients were biopsy-naïve, and in one patient the prebiopsy status was unknown. 865 biopsy cores were taken: 242 cores were from targeted biopsy and 623 cores from additional systematic biopsies.

PI-RADS scores were significantly higher in patients with PCa compared to patients without PCa $(4.4 \pm 0.7$ versus $3.2 \pm$ $0.4, p<0.001)$ and ADC values were significantly lower in tumor-positive lesions $\left(0.7 \pm 0.2\right.$ versus $0.8 \pm 0.210^{-3} \mathrm{~mm}^{2} / \mathrm{s}$, $p<0.05)$. Patients with PCa were significantly older $(69 \pm$ 8 versus $62 \pm 7$ years, $p<0.001)$ and prostate volume was significantly lower in the group with PCa $(60 \pm 40$ versus $84 \pm$ $52 \mathrm{ml}, p<0.05)$. No significant difference was found in PSA levels $(17 \pm 19$ versus $13 \pm 13 \mu \mathrm{g} / \mathrm{l})$, in lesion size $(18 \pm 7$ versus $14 \pm 9 \mathrm{~mm})$, or in BMI $\left(26 \pm 3\right.$ versus $\left.28 \pm 5 \mathrm{~kg} / \mathrm{m}^{2}\right)$.

In TP cohort, 59/75 (79\%) dominant lesions were located in the anterior prostate and 16/75 (21\%) were located in the posterior part of the organ. Concordantly, the PCa detection rate was significantly higher in the anterior gland $(44 / 56$, $79 \%)$ when compared to the posterior gland $(12 / 56,21 \%$; $p<0.001$, Table 2). The cancer detection rate, similar to the TR approach, when only considering the samples in the respective region, was comparable in the anterior gland $(44 / 59,75 \%)$ to the posterior gland (12/16, 75\%).

For this cohort, sensitivity and specificity of $86 \%$ and $84 \%$ were achieved for mpMRI with a Youden-selected cut-off value of PI-RADS 4, with an AUC of 0.89 (95\% CI: 0.8-1.0). NPV was $67 \%$ and PPV was $94 \%$.

In 54/56 patients with PCa, tumor diagnosis was obtained from targeted biopsy (Table 3 ). The cancer detection rate was improved from $72 \%$ to $75 \%$ when additional random biopsy cores were considered: in 2 patients (3\%), PCa was only found in the additional systematic biopsy cores (one patient with GS $3+3=6$ and one patient with GS $4+3=7 \mathrm{~b}$ ). A retrospective reanalysis of these patients revealed that in 1 of these 2 patients no suspicious lesion was detected at mpMRI in the region of $\mathrm{PCa}$ (GS $3+3=6$ ). In the other patient, MRI showed a suspicious lesion (PI-RADS score 5) in the documented region, indicating that MRI/US fusion-guided targeted biopsy failed (GS: $4+3=7 \mathrm{~b}$ ). Furthermore, in two patients (3\%), additional systematic biopsy cores resulted in a GS upgrade (one patient from GS $3+3=6$ to $3+4=7 \mathrm{a}$ and one patient from GS $4+4=8$ to $4+5=9$ ). A retrospective reanalysis showed that in one patient (GS upgrade from $3+3=6$ to $3+4=7$ a) the systematic biopsy core that revealed GS upgrade was taken from a region in the prostate which was close to the suspect lesion in MRI and potentially contained tissue of the index lesion. In the other patient, it is not reproducible from what region in the prostate was the systematic biopsy core with the upgrade retrieved; therefore no reason for upgrading can be identified.

A subgroup analysis of patients with PCa positive targeted biopsy ( $n=54$ ) revealed that in $44 / 54$ patients PCa was detected with the first biopsy core and in 10/54 patients with the second biopsy core and no additional PCa was found in the third targeted biopsy core. Thus, taking multiple biopsy cores from the target lesion improved cancer detection from
TABLE 4: Comparison of clinical parameters of transrectal (TR) and transperineal (TP) cohorts.

\begin{tabular}{lccc}
\hline $\begin{array}{l}\text { Clinical and MRI } \\
\text { parameters }\end{array}$ & $\begin{array}{c}\text { TR } \\
\text { Mean } \pm \text { SD }\end{array}$ & $\begin{array}{c}\text { TP } \\
\text { Mean } \pm \text { SD }\end{array}$ & $p$ value $^{*}$ \\
\hline Age (years) & $65 \pm 8$ & $67 \pm 8$ & $<0.05$ \\
PSA $(\mu \mathrm{g} / \mathrm{l})$ & $10 \pm 11$ & $16 \pm 18$ & $<0.05$ \\
Number of & $1.3 \pm 0.9(0-4)$ & $1.6 \pm 1.0(0-7)$ & $<0.05$ \\
prebiopsies & $58 \pm 31$ & $68 \pm 45$ & $\mathrm{~ns}$ \\
Prostate volume $(\mathrm{ml})$ & $27 \pm 4$ & $26 \pm 4$ & $\mathrm{~ns}$ \\
BMI $\left(\mathrm{kg} / \mathrm{m}^{2}\right)$ & $3 \pm 1$ & $4 \pm 1$ & $<0.001$ \\
PI-RADS & $0.9 \pm 0.3$ & $0.7 \pm 0.2$ & $<0.001$ \\
ADC $\left(10^{-3} \mathrm{~mm}^{2} / \mathrm{s}\right)$ & $13 \pm 5$ & $17 \pm 8$ & $<0.001$ \\
\hline Lesion size $(\mathrm{mm})$ &
\end{tabular}

${ }^{*}$ Unpaired $t$-test. PSA: prostate specific antigen; BMI: body mass index; ADC: apparent diffusion coefficient; ns: nonsignificant. ${ }^{* *}$ PI-RADS (overall score: 1-5): Prostate Imaging Reporting and Data System.

$59 \%$ with one biopsy core to $72 \%$ with two biopsy cores using the TP approach.

3.3. Comparison of Clinical Parameters between TR and TP Cohorts. Our TP cohort was significantly older $(67 \pm 8$ versus $65 \pm 8$ years, $p<0.05)$ and had more previous biopsies $(1.6 \pm 1.0$ versus $1.3 \pm 0.9, p<0.05)$ and higher PSA values $(16 \pm 19$ versus $10 \pm 11 \mu \mathrm{g} / \mathrm{l}, p<0.05)$ than patients in the TR cohort. Additionally, PI-RADS scores were higher $(4.1 \pm 0.7$ versus $3.4 \pm 1.1, p<0.001)$ and size of target lesions was larger $(17 \pm 7$ versus $13 \pm 5 \mathrm{~mm}, p<0.001)$ while ADC values were lower $\left(0.7 \pm 0.2\right.$ versus $\left.0.9 \pm 0.310^{-3} \mathrm{~mm}^{2} / \mathrm{s}, p<0.001\right)$ than those in our TR cohort. No significant difference in BMI was observed between the two groups ( $26 \pm 4$ versus $27 \pm 4$ ). Data are summarized in Table 4.

\section{Discussion}

We could demonstrate that mpMRI in combination with MRI/US fusion-guided biopsy yields high detection rates of PCa for both biopsy approaches. The BioJet system provides the opportunity to use both biopsy routes easily and can therefore offer different strategies for the individual patient with respect to the clinical setting. Additional untargeted, random biopsy cores as well as taking a second targeted biopsy core from the target lesion improved tumor detection rates in our study for both biopsy routes.

It is known that, in biopsy-naïve patients, detection rates for PCa with randomized untargeted biopsy are 20-30\% [2325] with decreasing rates of $10-20 \%$ for the second biopsy and $5-9 \%$ for the third biopsy $[25,26]$. However, there are also studies reporting a cancer detection rate of $41 \%$ with saturation biopsy (retrieving an average of 24 biopsy cores and more) in patients with previous negative biopsy [27].

In our study, we could achieve cancer detection rates of $39 \%$ for TR and $75 \%$ for TP combined targeted and systematic biopsies in a patient cohort with primarily prebiopsied patients (90\% with at least one previous biopsy), while less biopsy cores were needed than with saturation biopsy: $8 \pm 3$ 
cores for TR biopsy ( $3 \pm 1$ of which from targeted biopsy) and $12 \pm 1$ cores for TP biopsy ( $3 \pm 1$ from targeted biopsy) were taken.

When using radical prostatectomy tissue as reference, it has been reported that significant PCa might be missed in $8-24 \%$ of patients by mpMRI [28-30]. Cancer detection rate in our study was improved from 33\% to $39 \%$ with TR biopsy and from $72 \%$ to $75 \%$ with TP biopsy when additional untargeted biopsy cores were considered. It has been stated earlier that the combination of systematic and targeted biopsy schemes provides the highest detection rate [10] and our results go along with that. Nevertheless, in our study, 4 patients (2.6\%) showed suspect lesions on MRI but negative results from targeted biopsy, while untargeted biopsy revealed PCa. It can be assumed that either MRI/US fusion or fusion-targeted biopsy has failed in these cases. However, $2.6 \%$ in total seems to be a justifiable error with the technique, providing that patients are correctly informed in advance.

Furthermore, evidence suggested that, in MRI/US fusionguided biopsy, a two-core biopsy should be performed per target lesion [10,31], but clear recommendations are still missing. However, a recent study found limited benefit when taking a second biopsy core per target lesion with MRI inbore biopsy [17]. In our TR and TP cohorts, we found PCa diagnosed with an additional targeted biopsy core in about $20-30 \%$ of cases, indicating that at least two targeted biopsy cores might be reasonable. A prospective trial would be necessary to elucidate that very important issue.

With the descriptive design of our study (not randomized, different starting points of TR and TP approach, selection bias), comparing the two cohorts is not intended. We could show that, with one software system, both biopsy routes are feasible and equally effective. Our two cohorts differ in some aspects. In 2012, we started using mpMRI and MRI/US fusion-guided biopsy and procedures (MRI protocol, workflow, and reporting) were not standardized due to a lack of recommendations about when to use MRI fusion-guided biopsy in men with clinical suspicion for PCa. Between July 2012 and January 2015, only TR fusion-guided biopsy ( $n=$ 62) was performed and TP biopsy was introduced in our institution in February 2015. Initial uncertainty about NPV of mpMRI as well as missing recommendation about cutoff values of PI-RADS scores led to higher indication rates of targeted biopsy, even with lower PI-RADS scores in the initial phase of MRI/US fusion-guided biopsy, when we only used the TR biopsy route. This could be an explanation for our finding that, in our patients that underwent TP biopsy (at later time points after February 2015), PI-RADS scores and lesion size were significantly higher and ADC values were significantly lower than those in our patients that underwent TR biopsy predominantly before February 2015. Furthermore, there was an ongoing optimization of MRI protocol and improvement of workflow, personal learning curve in MRI interpretation, and biopsy planning and biopsy performance. An existing learning curve for prostate MRI interpretation has been described [32-34]. Gaziev et al. recently reported that cancer detection rate improved within two years from $42 \%$ to $81 \%$, sensitivity/specificity improved from $93 / 9 \%$ to $85 / 52 \%$, and NPV improved from $67 \%$ to $89 \%$ [35].
In our study, one anterior lesion could not be reached with TR approach. Therefore, TP biopsy could be helpful to reach the anterior part of the prostate. In our patient cohort, anterior lesions were more often biopsied with the TP approach. Notably, when comparing the yield of PCa detection within the two cohorts, the percentage of detected PCa located in the anterior half of the prostate was related to the percentage of MRI target lesions in the anterior prostate (Table 2). Therefore, the observation of a higher absolute number of anteriorly located PCa in the TP cohort is solely due to the higher absolute number of anteriorly located MRI target lesions and not from a higher detection rate for anteriorly located lesions compared to posteriorly located lesions with the respective biopsy approach.

Previous studies suggested that TR biopsy might hold a higher risk of infection, since faecal bacteria can enter blood circulation after retrieving specimen from the prostate [36]. Particularly in men with higher risks of infection or rectal bleeding (e.g., with hemorrhoids), complications might be avoided with the TP biopsy method. Pain management, on the other hand, is more challenging with TP biopsy, since it has been reported to be more painful [37]. At our institution, TP biopsy is only performed in general anesthesia and therefore might not be suitable for outpatient procedures, although there are few working groups reporting the possibility to obtain it under local anesthesia [38].

Limitations of our study are the retrospective design and solely descriptive analysis of TR or TP biopsy approach in a clinical setting. Patients were not randomized into TR and TP cohort. The decision for one of the two biopsy routes depended on a doctors-patient shared decision, under consideration of patients' preference, prostate size, lesion localization, and technical availability. And, finally, the TP approach has been available since February 2015, which introduced a bias.

\section{Conclusion}

In conclusion, we demonstrate that MRI/US fusion-guided biopsy has high accuracy for the detection of PCa with both TR and TP approaches. Our biopsy system provides the opportunity to offer both biopsy routes to patients at risk of $\mathrm{PCa}$ and to adjust diagnostic strategy to the individual clinical setting. In addition, the TP approach gives the options for fusion-guided focal therapy strategies.

\section{Abbreviations}

ACR: American College of Radiology

ADC: Apparent diffusion coefficient

AFS: Anterior fibromuscular stroma

AUC: Area under the curve

DCE: Dynamic contrast-enhanced imaging

DWI: Diffusion-weighted imaging

ESUR: European Society of Urogenital Radiology

GS: $\quad$ Gleason score

mpMRI: Multiparametric magnetic resonance imaging

NPV: Negative predictive value

PCa: Prostate cancer 


$\begin{array}{ll}\text { PI-RADS: } & \text { Prostate Imaging Reporting and Data System } \\ \text { PPV: } & \text { Positive predictive value } \\ \text { PSA: } & \text { Prostate specific antigen } \\ \text { SD: } & \text { Standard deviation } \\ \text { TP: } & \text { Transperineal } \\ \text { TR: } & \text { Transrectal } \\ \text { TSE: } & \text { Turbo spin echo } \\ \text { US: } & \text { Ultrasound. }\end{array}$

\section{Conflicts of Interest}

The authors declare that there are no conflicts of interest regarding the publication of this article.

\section{Authors' Contributions}

Susanne Tewes and Inga Peters contributed equally to this work.

\section{Acknowledgments}

Katja Hueper and Susanne Tewes received funding from the Junge Akademie Program, Hannover Medical School.

\section{References}

[1] M. de Rooij, E. H. J. Hamoen, J. J. Fütterer, J. O. Barentsz, and M. M. Rovers, "Accuracy of multiparametric MRI for prostate cancer detection: a meta-analysis," American Journal of Roentgenology, vol. 202, no. 2, pp. 343-351, 2014.

[2] L. Dickinson, H. U. Ahmed, C. Allen et al., "Magnetic resonance imaging for the detection, localisation, and characterisation of prostate cancer: recommendations from a European consensus meeting," European Urology, vol. 59, no. 4, pp. 477-494, 2011.

[3] F. Bratan, E. Niaf, C. Melodelima et al., "Influence of imaging and histological factors on prostate cancer detection and localisation on multiparametric MRI: a prospective study," European Radiology, vol. 23, no. 7, pp. 2019-2029, 2013.

[4] T. Franz, J. von Hardenberg, A. Blana et al., "MRI/TRUS fusionguided prostate biopsy: value in the context of focal therapy," Der Urologe A, vol. 56, no. 2, pp. 208-216, 2017.

[5] C. M. Moore, N. L. Robertson, N. Arsanious et al., "Imageguided prostate biopsy using magnetic resonance imagingderived targets: a systematic review," European Urology, vol. 63, no. 1, pp. 125-140, 2013.

[6] J. P. Radtke, T. H. Kuru, S. Boxler et al., "Comparative Analysis of transperineal template saturation prostate biopsy versus magnetic resonance imaging targeted biopsy with magnetic resonance imaging-ultrasound fusion guidance," The Journal of Urology, vol. 193, no. 1, pp. 87-94, 2015.

[7] M. Valerio, I. Donaldson, M. Emberton et al., "Detection of clinically significant prostate cancer using magnetic resonance imaging-ultrasound fusion targeted biopsy: a systematic review," European Urology, vol. 68, no. 1, pp. 8-19, 2015.

[8] I. G. Schoots, M. J. Roobol, D. Nieboer, C. Bangma, E. Steyerberg, and M. Hunink, "Magnetic resonance imaging-targeted biopsy may enhance the diagnostic accuracy of significant prostate cancer detection compared to standard transrectal ultrasound-guided biopsy: a systematic review and metaanalysis," European Urology, vol. 68, no. 3, pp. 438-450, 2015.
[9] M. M. Siddiqui, S. Rais-Bahrami, B. Turkbey et al., "Comparison of MR/ultrasound fusion-guided biopsy with ultrasoundguided biopsy for the diagnosis of prostate cancer," The Journal of the American Medical Association, vol. 313, no. 4, pp. 390-397, 2015.

[10] A. van Hove, P.-H. Savoie, C. Maurin et al., "Comparison of image-guided targeted biopsies versus systematic randomized biopsies in the detection of prostate cancer: a systematic literature review of well-designed studies," World Journal of Urology, vol. 32, no. 4, pp. 847-858, 2014.

[11] N. Mottet, J. Bellmunt, M. Bolla et al., "EAU-ESTRO-SIOG guidelines on prostate cancer. Part 1: screening, diagnosis, and local treatment with curative intent," European Urology, vol. 71, no. 4, pp. 618-629, 2017.

[12] Leitlinienprogramm_Onkologie, Interdisziplinäre Leitlinie der Qualität S3 zur Früherkennung, Diagnose und Therapie der verschiedenen Stadien des Prostatakarzinoms, 2014.

[13] L. Marks, S. Young, and S. Natarajan, "MRI-ultrasound fusion for guidance of targeted prostate biopsy," Current Opinion in Urology, vol. 23, no. 1, pp. 43-50, 2013.

[14] R. Hara, Y. Jo, T. Fujii et al., "Optimal approach for prostate cancer detection as initial biopsy: prospective randomized study comparing transperineal versus transrectal systematic 12core biopsy," Urology, vol. 71, no. 2, pp. 191-195, 2008.

[15] A. Takenaka, R. Hara, T. Ishimura et al., "A prospective randomized comparison of diagnostic efficacy between transperineal and transrectal 12-core prostate biopsy," Prostate Cancer and Prostatic Diseases, vol. 11, no. 2, pp. 134-138, 2008.

[16] H. Huang, W. Wang, T. Lin et al., "Comparison of the complications of traditional 12 cores transrectal prostate biopsy with image fusion guided transperineal prostate biopsy," $B M C$ Urology, vol. 16, no. 1, article 68, 2016.

[17] L. Schimmoller, M. Quentin, D. Blondin et al., "Targeted MRIguided prostate biopsy: are two biopsy cores per MRI-lesion required?" European Radiology, vol. 26, no. 11, pp. 3858-3864, 2016.

[18] J. O. Barentsz, J. Richenberg, R. Clements et al., "ESUR prostate MR guidelines 2012," European Radiology, vol. 22, no. 4, pp. 746757, 2012.

[19] J. C. Weinreb, J. O. Barentsz, P. L. Choyke et al., "PI-RADS prostate imaging - reporting and data system: 2015, version 2," European Urology, vol. 69, no. 1, pp. 16-40, 2015.

[20] S. Shoji, S. Hiraiwa, J. Endo et al., "Manually controlled targeted prostate biopsy with real-time fusion imaging of multiparametric magnetic resonance imaging and transrectal ultrasound: An early experience," International Journal of Urology, vol. 22, no. 2, pp. 173-178, 2015.

[21] S. Tewes, K. Hueper, D. Hartung et al., "Targeted MRI/TRUS fusion-guided biopsy in men with previous prostate biopsies using a novel registration software and multiparametric MRI PI-RADS scores: first results," World Journal of Urology, vol. 33, no. 11, pp. 1707-1714, 2015.

[22] A. V. D’Amico, R. Whittington, S. Bruce Malkowicz et al., "Biochemical outcome after radical prostatectomy, external beam radiation therapy, or interstitial radiation therapy for clinically localized prostate cancer," Journal of the American Medical Association, vol. 280, no. 11, pp. 969-974, 1998.

[23] O. Ukimura, J. A. Coleman, A. de la Taille et al., "Contemporary role of systematic prostate biopsies: indications, techniques, and implications for patient care," European Urology, vol. 63, no. 2, pp. 214-230, 2013.

[24] S. F. Shariat and C. G. Roehrborn, "Using biopsy to detect prostate cancer," Reviews in Urology, vol. 10, no. 4, pp. 262-280, 2008. 
[25] B. Djavan, V. Ravery, A. Zlotta et al., "Prospective evaluation of prostate cancer detected on biopsies 1, 2, 3 and 4: when should we stop?" The Journal of Urology, vol. 166, no. 5, pp. 1679-1683, 2001.

[26] C. G. Roehrborn, G. J. Pickens, and J. S. Sanders, "Diagnostic yield of repeated transrectal ultrasound-guided biopsies stratified by specific histopathologic diagnoses and prostate-specific antigen levels," Urology, vol. 47, no. 3, pp. 347-352, 1996.

[27] J. Walz, M. Graefen, F. K.-H. Chun et al., "High incidence of prostate cancer detected by saturation biopsy after previous negative biopsy series," European Urology, vol. 50, no. 3, pp. 498505, 2006.

[28] J. P. Radtke, C. Schwab, M. B. Wolf et al., "Multiparametric Magnetic Resonance Imaging (MRI) and MRI-transrectal ultrasound fusion biopsy for index tumor detection: correlation with radical prostatectomy specimen," European Urology, vol. 70, no. 5, pp. 846-853, 2016.

[29] J. D. Le, N. Tan, E. Shkolyar et al., "Multifocality and prostate cancer detection by multiparametric magnetic resonance imaging: Correlation with whole-mount histopathology," European Urology, vol. 67, no. 3, pp. 569-576, 2015.

[30] N. Tan, D. J. Margolis, D. Y. Lu et al., "Characteristics of detected and missed prostate cancer foci on 3-T multiparametric MRI using an endorectal coil correlated with whole-mount thinsection histopathology," American Journal of Roentgenology, vol. 205, no. 1, pp. W87-W92, 2015.

[31] E. Baco, E. Rud, L. M. Eri et al., "A randomized controlled trial to assess and compare the outcomes of two-core prostate biopsy guided by fused magnetic resonance and transrectal ultrasound images and traditional 12-core systematic biopsy," European Urology, vol. 69, no. 1, pp. 149-156, 2016.

[32] O. Akin, C. C. Riedl, N. M. Ishill, C. S. Moskowitz, J. Zhang, and H. Hricak, "Interactive dedicated training curriculum improves accuracy in the interpretation of MR imaging of prostate cancer," European Radiology, vol. 20, no. 4, pp. 995-1002, 2010.

[33] A. P. S. Kirkham, P. Haslam, J. Y. Keanie et al., "Prostate MRI: Who, when, and how? Report from a UK consensus meeting," Clinical Radiology, vol. 68, no. 10, pp. 1016-1023, 2013.

[34] K. C. Latchamsetty, L. S. Borden Jr., C. R. Porter et al., "Experience improves staging accuracy of endorectal magnetic resonance imaging in prostate cancer: what is the learning curve?" The Canadian Journal of Urology, vol. 14, no. 1, pp. 34293434, 2007.

[35] G. Gaziev, K. Wadhwa, T. Barrett et al., "Defining the learning curve for multiparametric magnetic resonance imaging (MRI) of the prostate using MRI-transrectal ultrasonography (TRUS) fusion-guided transperineal prostate biopsies as a validation tool," BJU International, vol. 117, no. 1, pp. 80-86, 2016.

[36] D. Steensels, K. Slabbaert, L. De Wever, P. Vermeersch, H. Van Poppel, and J. Verhaegen, "Fluoroquinolone-resistant E. coli in intestinal flora of patients undergoing transrectal ultrasoundguided prostate biopsy-should we reassess our practices for antibiotic prophylaxis?" Clinical Microbiology and Infection, vol. 18, no. 6, pp. 575-581, 2012.

[37] L.-H. Guo, R. Wu, H.-X. Xu et al., "Comparison between ultrasound guided transperineal and transrectal prostate biopsy: a prospective, randomized, and controlled trial," Scientific Reports, vol. 5, Article ID 16089, 2015.

[38] J. B. Smith, R. Popert, M. C. Nuttall, L. Vyas, J. Kinsella, and D. Cahill, "Transperineal sector prostate biopsies: A local anesthetic outpatient technique," Urology, vol. 83, no. 6, pp. 1344-1349, 2014. 


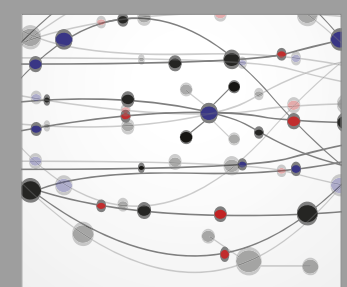

The Scientific World Journal
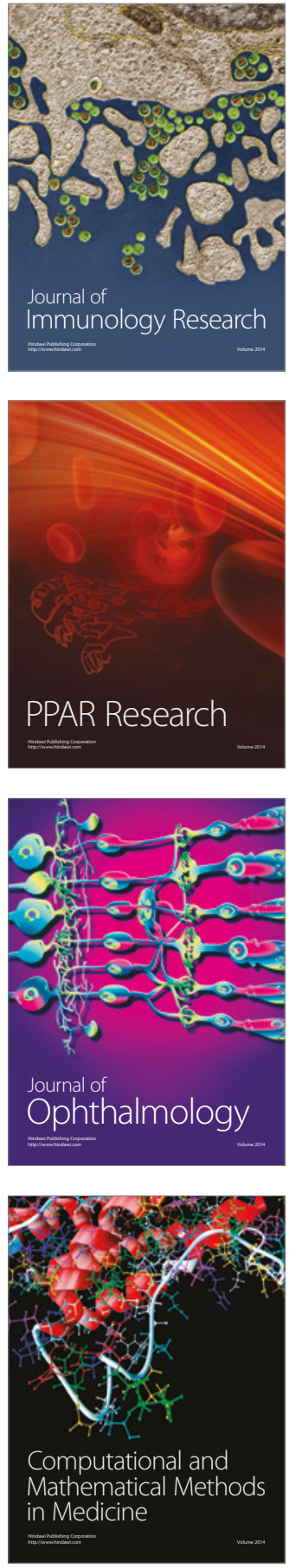

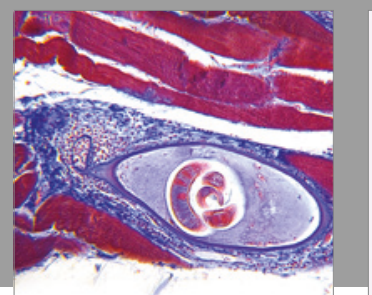

Gastroenterology Research and Practice
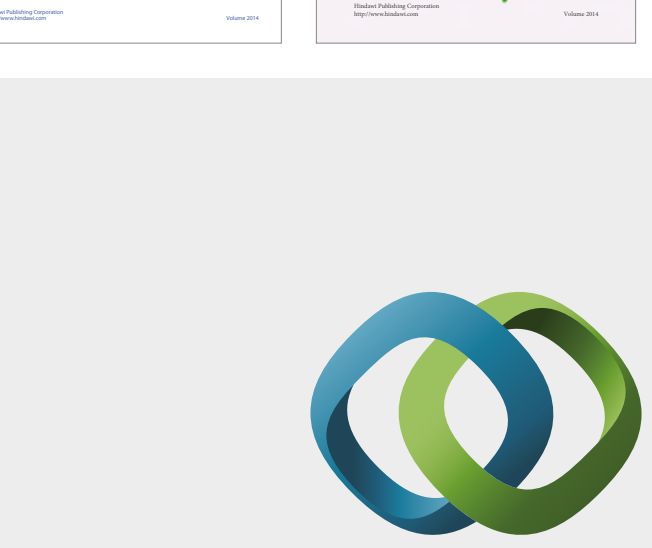

\section{Hindawi}

Submit your manuscripts at

https://www.hindawi.com
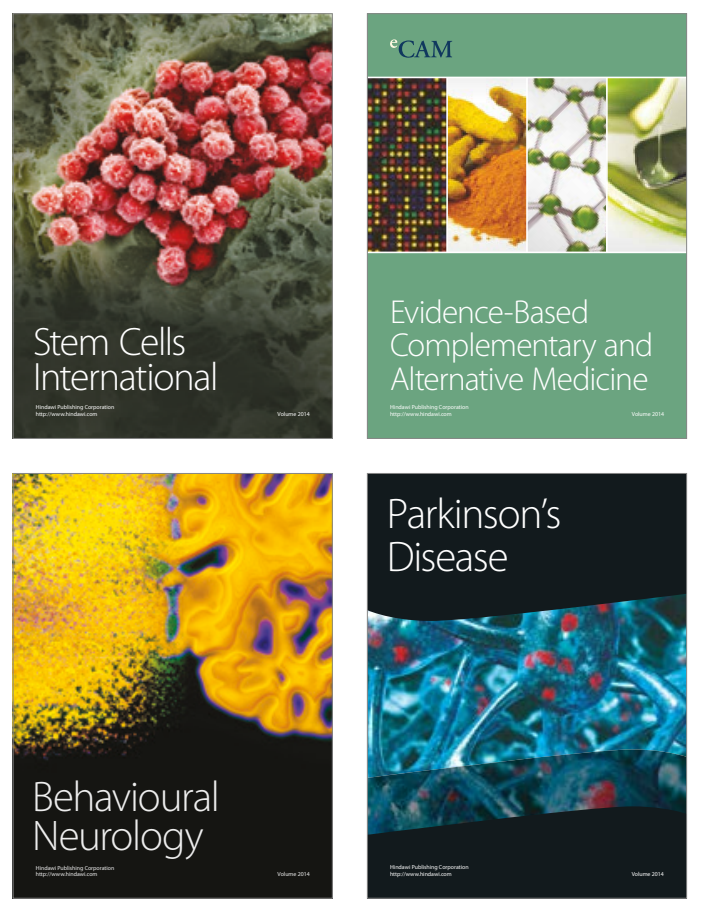
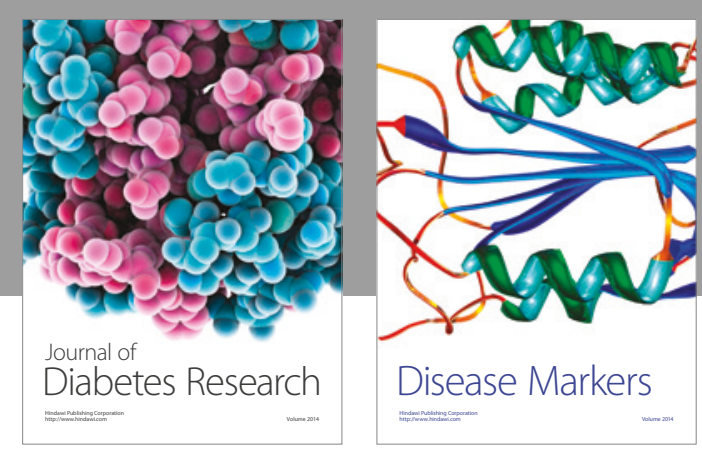

Disease Markers
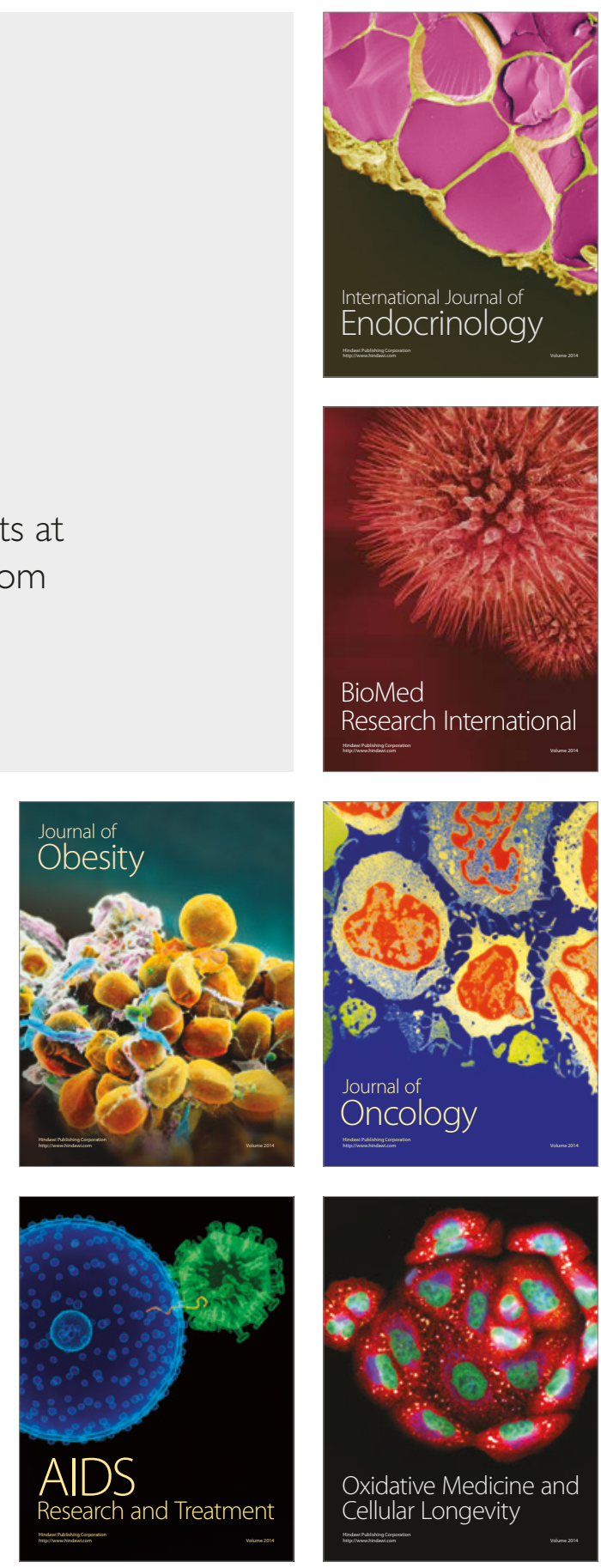